OPEN ACCESS

Edited by:

Miklos Fuzi,

Semme/weis University, Hungary

Reviewed by:

Carmen Losasso,

Istituto Zooprofilattico Sperimentale

delle Venezie, Italy

Javad Sharifi-Rad,

Shahid Beheshti University of Medical

Sciences, Iran

Fernando Rogerio Pavan, Universidade Estadual Paulista Júlio de Mesquita Filho (UNESP), Brazil

*Correspondence:

José A. Ainsa

ainsa@unizar.es

Adela G. de la Campa

agcampa@isciii.es

${ }^{\dagger}$ Present address:

María T. García,

Departamento de Microbiología II,

Facultad de Biología, Universidad

Complutense de Madrid, Madrid,

Spain

Liliana Rodrigues,

Unit of Medical Microbiology, Global

Health and Tropical Medicine, Instituto de Higiene e Medicina Tropical, Universidade Nova de Lisboa, Lisbon,

Portugal

Specialty section: This article was submitted to Antimicrobials, Resistance and Chemotherapy,

a section of the journal Frontiers in Microbiology

Received: 22 March 2018 Accepted: 04 July 2018 Published: 24 July 2018

Citation:

García MT, Carreño D, Tirado-Vélez JM, Ferrándiz MJ, Rodrigues L, Gracia B, Amblar M, Ainsa JA and de la Campa AG (2018) Boldine-Derived Alkaloids Inhibit the Activity of DNA Topoisomerase and Growth of Mycobacterium tuberculosis. Front. Microbiol. 9:1659. doi: 10.3389/fmicb.2018.01659

\section{Boldine-Derived Alkaloids Inhibit the Activity of DNA Topoisomerase I and Growth of Mycobacterium tuberculosis}

\author{
María T. García1†, David Carreñoํㅜ, José M. Tirado-Vélez¹, María J. Ferrándiz, \\ Liliana Rodrigues $2,3,4+$, Begoña Gracia ${ }^{2,3}$, Mónica Amblar ${ }^{5}$, José A. Ainsa ${ }^{2,3 *}$ and \\ Adela G. de la Campa ${ }^{1,6 *}$
}

1 Unidad de Genética Bacteriana, Centro Nacional de Microbiología, Instituto de Salud Carlos III, Madrid, Spain, ${ }^{2}$ CIBER de Enfermedades Respiratorias, Madrid, Spain, ${ }^{3}$ Departamento de Microbiología, Medicina Preventiva y Salud Pública, Facultad de Medicina, Universidad de Zaragoza, Zaragoza, Spain, ${ }^{4}$ Fundación Agencia Aragonesa para la Investigación y el Desarrollo, Zaragoza, Spain, ${ }^{5}$ Unidad de Patología Molecular de Neumococo, Centro Nacional de Microbiología, Instituto de Salud Carlos III, Madrid, Spain, ${ }^{6}$ Presidencia, Consejo Superior de Investigaciones Cientificas, Madrid, Spain

The spread of multidrug-resistant isolates of Mycobacterium tuberculosis requires the discovery of new drugs directed to new targets. In this study, we investigated the activity of two boldine-derived alkaloids, seconeolitsine (SCN) and $\mathrm{N}$-methyl-seconeolitsine ( $\mathrm{N}$ SCN), against $M$. tuberculosis. These compounds have been shown to target DNA topoisomerase I enzyme and inhibit growth of Streptococcus pneumoniae. Both SCN and $N$-SCN inhibited $M$. tuberculosis growth at 1.95-15.6 $\mu \mathrm{M}$, depending on the strain. In $M$. smegmatis this inhibitory effect correlated with the amount of topoisomerase I in the cell, hence demonstrating that this enzyme is the target for these alkaloids in mycobacteria. The gene coding for topoisomerase I of strain H37Rv (MtbTopol) was cloned into PQE1 plasmid of Escherichia coli. MtbTopol was overexpressed with an N-terminal 6-His-tag and purified by affinity chromatography. In vitro inhibition of MtbTopol activity by SCN and N-SCN was tested using a plasmid relaxation assay. Both SCN and N-SCN inhibited $50 \%$ of the enzymatic activity at 5.6 and $8.4 \mu \mathrm{M}$, respectively. Cleavage of single-stranded DNA was also inhibited with SCN. The effects on DNA supercoiling were also evaluated in vivo in plasmid-containing cultures of M. tuberculosis. Plasmid supercoiling densities were -0.060 in cells untreated or treated with boldine, and -0.072 in $1 \times$ MIC N-SCN treated cells, respectively, indicating that the plasmid became hypernegatively supercoiled in the presence of $\mathrm{N}-\mathrm{SCN}$. Altogether, these results demonstrate that the $M$. tuberculosis topoisomerase I enzyme is an attractive drug target, and that SCN and N-SCN are promising lead compounds for drug development.

Keywords: Mycobacterium tuberculosis, DNA topoisomerase I inhibitor, DNA supercoiling, N-methylseconeolitsine, seconeolitsine, antituberculosis activity, drug discovery

\section{INTRODUCTION}

Mycobacterium tuberculosis is the causative agent of tuberculosis (TB), a major global health issue. In 2016, an estimated 6.3 million people developed TB, 1.3 million of HIV-negative people and 374,000 HIV-positive people died because of TB (World Health Organization, 2017). Nowadays, the recommended treatment for drug-susceptible TB is a 6-month regime of four first-line drugs: 
isoniazid, rifampicin, ethambutol, and pyrazinamide. Failures in drug supply and patients' lack of adherence to treatment (among other factors) have resulted in the emergence of resistance to anti-TB drugs. Multidrug-resistant TB (MDR-TB) is characterized by resistance to both rifampicin and isoniazid. Treatment of these strains takes longer and requires additional drugs that are more toxic and less effective. In fact, in 2016, a total of 490,000 people developed MDR-TB globally, leading to an estimated 240,000 deaths. In addition, an estimated $6.2 \%$ of MDR-TB cases progressed into extensively drug-resistant TB (XDR-TB), defined as MDR-TB with additional resistance to a fluoroquinolone and at least one of three injectable second-line drugs (amikacin, kanamycin, or capreomycin).

Given these facts, new anti-TB drugs are urgently needed. Rifampicin, the most effective anti-TB drug, was introduced into clinical practice in the 1960s. Since then only one drug has been developed, bedaquiline, which recently was approved for MDRTB treatment (Palomino and Martín, 2013). Even though when new drug candidates are emerging from the pipeline, and some are undergoing clinical trials (Zumla et al., 2014), investigation of new anti-TB drugs needs to continue. In order to avoid crossresistance with already existing drugs, it is necessary to identify and characterize new targets for anti-TB drugs (Sharifi-Rad et al., 2017).

The DNA supercoiling level is an essential parameter of bacteria, given that it is a critical component of DNA replication, transcription, and recombination (Champoux, 2001). An adequate level of DNA supercoiling is maintained by DNA topoisomerase enzymes. These enzymes act on double-strand DNA, cleaving either both strands (type II enzymes) or one of the DNA strands (type I enzymes) allowing the intact segment to pass through. The cleaved DNA is then resealed before being released. DNA topoisomerase I (TopoI) has been proposed as a new antibacterial target (Tse-Dinh, 2009). Some natural compounds inhibited in vitro the enzymatic activity of this enzyme from Yersinia pestis and Escherichia coli, and were shown to enhance DNA cleavage (Cheng et al., 2007, 2013). Among them was a phenanthrene alkaloid able to inhibit the relaxation activity of E. coli TopoI, although no significant inhibition in cell growth was observed (Cheng et al., 2007). We have established TopoI as a new drug target in Streptococcus pneumoniae and described two novel alkaloid compounds: seconeolitsine (SCN) and $N$-methyl-seconeolitsine (N-SCN), derived from boldine. Both compounds inhibited S. pneumoniae TopoI activity in vitro at concentrations equivalent to those necessary to inhibit bacterial growth $(\sim 10 \mu \mathrm{M})$ without affecting human cell viability (García et al., 2011). M. tuberculosis possess two DNA topoisomerases: one type II enzyme, DNA gyrase, which is targeted by fluoroquinolone antibiotics (Kumar et al., 2014) and one type I enzyme, topoisomerase I (MtbTopoI), which is encoded by Rv3646c (topA) gene. This gene has been shown to be essential for M. tuberculosis growth (Kumar et al., 2014).

The aim of the present study was to investigate these two boldine-derivative alkaloids as potential inhibitors of the MtbTopoI enzyme, a scarcely explored drug target. SCN and $N$-SCN inhibited growth of $M$. tuberculosis at relatively low concentrations and also inhibited MtbTopoI activity in vitro. In addition, their effects on DNA supercoiling were evaluated in plasmid-containing cultures of $M$. tuberculosis.

\section{MATERIALS AND METHODS}

\section{Bacterial Strains, Growth Conditions and Determination of Minimal Inhibitory Concentrations (MICs)}

The reference laboratory strain M. tuberculosis H37Rv (ATCC 25618) and a panel of eight genetically distinct clinical strains of $M$. tuberculosis were used for drug susceptibility testing. This included strain GC1237, a highly transmissible strain of the Beijing lineage. A derivative of the $\mathrm{H} 37 \mathrm{Rv}$ strain containing plasmid vector pSUM36 (Ainsa et al., 1996) was used for testing the in vivo effect of alkaloids on DNA supercoiling. To determine the mechanism of action of topoisomerase inhibitors, Mycobacterium smegmatis mc $^{2} 155$ (Snapper et al., 1990) was used along with its derivative MsPptrtopoI conditional knock-down mutant (Ahmed et al., 2015), in which levels of topoisomerase I (MsTopoI) can be reduced by addition of anhydrotetracycline (ATc). All strains were grown in Middlebrook 7H9 broth (Becton Dickinson) supplemented with 10\% ADC (Becton Dickinson) and $0.05 \%$ Tween 80 (Sigma). Kanamycin ( $50 \mathrm{mg} / \mathrm{L}$ ) was added to ensure the maintenance of plasmid pSUM36. Minimal inhibitory concentrations (MICs) were determined by microdilution as previously reported for M. tuberculosis (Palomino et al., 2002); MICs of drugs for $M$. smegmatis were determined by the same method except that plates were incubated for 3 days. The MIC was defined as the lowest concentration of drug that prevented change of resazurin from its oxidized form (blue) into the reduced one (pink), which is indicative of bacterial growth. Imipramine, a well-known topoisomerasepoison described previously (Godbole et al., 2015) was included as a control. For the time-kill kinetics experiments, a bacterial inoculum of $10^{7} \mathrm{CFU} / \mathrm{ml}$ was incubated in the presence of inhibitory concentrations of $\mathrm{N}$-SCN $(8 \times \mathrm{MIC})$ for $24 \mathrm{~h}$ at $37^{\circ} \mathrm{C}$; then, the culture was serially diluted in order to determine the number of viable bacteria, by plating on Middlebrook 7H10 plates; relative CFUs in comparison with untreated control cultures were determined.

\section{Cloning and Expression of M. tuberculosis topA in Escherichia coli}

The Rv3646c (topA) gene from M. tuberculosis was amplified by PCR using $0.5 \mu \mathrm{g}$ of chromosomal DNA from $M$. tuberculosis $\mathrm{H} 37 \mathrm{Rv}$ strain as a template and $1 \mu \mathrm{M}$ each of the following synthetic oligonucleotide $5^{\prime}$-phosphorylated primers: TopATbUP2 (5' -ATGGCTGACCCGAAAACGAAGGG-3') and TopATbDOWN2 (5'-cgcgcgcatgcCTAGTCGCGCTTGGCTGCC-3'). The latter including a PaeI restriction site (lowercase underlined). The sequence of primer TopATbUP2 contained the ATG initiation codon and the sequence of TopATbDOWN contained the TAG stop codon of Rv3646 (both upper-case underlined). Amplification was achieved using an initial cycle of $2 \mathrm{~min}$ denaturation at $94^{\circ} \mathrm{C}$, followed by $30 \mathrm{~s}$ annealing at $55^{\circ} \mathrm{C}$, and 
a 3 min polymerase extension with Platinum Taq Polymerase high-fidelity (Invitrogen) at $68^{\circ} \mathrm{C}$. This was followed by 34 cycles of $30 \mathrm{~s}$ at $94^{\circ} \mathrm{C}, 30 \mathrm{~s}$ at $55^{\circ} \mathrm{C}$ and $3 \mathrm{~min}$ at $68^{\circ} \mathrm{C}$ followed by slow cooling at $8^{\circ} \mathrm{C}$. After completing amplification, excess oligonucleotides were removed using the QIAquick PCR Purification Kit (QIAGEN). The amplicon was digested with PaeI, cloned into plasmid vector pQE1 (TAGZyme, Qiagen) digested with PaeI + PvuII (blunt ends), resulting in recombinant plasmid $\mathrm{PQE}-\mathrm{Mtb}$ TopA that was introduced into E. coli XL1Blue. Sequencing with oligonucleotides pQE-seq3 $\left(5^{\prime}\right.$-AGCTAGCTTGGATTCTCACC-3') and pQE-seq5 (5'-GA GGCCCTTTCGTCTTCA-3') was performed to confirm that no mutation was introduced during cloning. The $\mathrm{pQE} 1$ vector/XL1 host cloning system permits the hyperproduction of $6 \times$ Histagged recombinant proteins encoded by genes placed under the control of a phage T5 promoter and two lac operator sequences. A culture of E. coli XL1/pQE-MtbTopA was grown at $37^{\circ} \mathrm{C}$ in $2 \mathrm{~L}$ of LB medium containing $100 \mu \mathrm{g} / \mathrm{ml}$ of ampicillin until it reached an $\mathrm{OD}_{620}$ of 0.6 . Cells were induced with $1 \mathrm{mM}$ Isopropyl- $\beta-\mathrm{D}-$ thiogalactoside (IPTG) for $3 \mathrm{~h}$, harvested by centrifugation and suspended in $50 \mathrm{ml}$ of buffer $\mathrm{A}\left(50 \mathrm{mM} \mathrm{NaH}_{2} \mathrm{PO}_{4} \mathrm{pH} 8,300 \mathrm{mM}\right.$ $\mathrm{NaCl}, 1 \mathrm{mM}$ PMSF) containing $10 \mathrm{mM}$ imidazole. Triton X100 was added to a final concentration of $0.25 \%$, and the solution was frozen at $-80^{\circ} \mathrm{C}$ then defrosted on ice. Cells were then incubated with lysozyme $(1 \mathrm{mg} / \mathrm{ml})$ at $4^{\circ} \mathrm{C}$ for $60 \mathrm{~min}$ with lysozyme and sonicated for $100 \mathrm{~s}$ ( 5 times for $20 \mathrm{~s}$, with 1 min cooling between each burst) in a Sonifier B-12 (Branson Co., Connecticut). The crude extract was centrifuged at $10.000 \times g$ for $20 \mathrm{~min}$ and the resulting supernatant filtered using a $0.45 \mu \mathrm{m}$ Millipore Millex ${ }^{\circledR} \mathrm{HA}$ filter. The MtbTopoI protein was purified by affinity chromatography in $1 \mathrm{ml} \mathrm{Ni-NTA} \mathrm{(QIAGEN)} \mathrm{column} \mathrm{attached}$ to an ÄKTA FPLC system using the standard manufacturer protocol for this column. The column was equilibrated with 10 column volumes of buffer A. Non-specifically bound proteins were removed with a 20 column volume of $10-60 \mathrm{mM}$ imidazole in buffer A. Specifically bound proteins were eluted with a 40 column volume gradient of 60-350 mM imidazole. Fractions of $2 \mathrm{ml}$ were collected and analyzed by SDS-10\% polyacrylamide gel electrophoresis followed by Coomassie blue staining. Fractions containing a protein of the expected size, which had eluted at around $100 \mathrm{mM}$ imidazole were dialyzed against buffer $\mathrm{B}(50 \mathrm{mM}$ $\mathrm{NaH}_{2} \mathrm{PO}_{4} \mathrm{pH} 8,100 \mathrm{mM} \mathrm{NaCl}, 0.5 \mathrm{mM}$ DTT, 50\% glycerol). The purified $\mathrm{Mtb}$ TopoI protein was stored at $-20^{\circ} \mathrm{C}$ and thereby remained active for at least 12 months.

\section{Relaxation of pBR322 in Vitro by M. tuberculosis Topol and Cleavage Assay}

Reactions were carried out using $0.5 \mu \mathrm{g}$ of supercoiled plasmid pBR322 in $200 \mu \mathrm{l}$ of a buffer containing $50 \mathrm{mM}$ Tris $\mathrm{HCl} \mathrm{pH} \mathrm{8,}$ $20 \mathrm{mM} \mathrm{KCl}, 10 \mathrm{mM} \mathrm{MgCl}, 0.5 \mathrm{mM}$ DTT and $30 \mu \mathrm{g} \mathrm{BSA} / \mathrm{ml}$. After $1 \mathrm{~h}$ incubation at $37^{\circ} \mathrm{C}$ in the presence on MtbTopoI, the reaction was terminated by incubation at $37^{\circ} \mathrm{C}$ for 2 min after adding EDTA to a final concentration of $50 \mathrm{mM}$ followed by an incubation of $1 \mathrm{~h}$ at $37^{\circ} \mathrm{C}$ in the presence of $1 \%$ SDS and $100 \mu \mathrm{g} / \mathrm{ml}$ proteinase $\mathrm{K}$. When required, samples were ethanol precipitated and suspended in $\mathrm{H}_{2} \mathrm{O}$. Reaction products were analyzed by electrophoresis in $1.2 \%$ agarose gels in $1 \times$ TAE at $18 \mathrm{~V}$ for $16 \mathrm{~h}$. In order to test the effect of drugs against the MtbTopoI-mediated relaxation of pBR322, the enzyme was pre-incubated with these compounds for $10 \mathrm{~min}$ at $4^{\circ} \mathrm{C}$ in a final volume of $20 \mu \mathrm{l}$. Electrophoresis was carried out in gels in the presence and absence of ethidium bromide (EtBr). Quantification of DNA was performed by densitometers of EtBr stained agarose gels (Quantity One, Bio-Rad Laboratories). To calculate MtbTopoI activity, the amount of the CCC form was measured and the value divided by the total amount of DNA in each well. The mean $\mathrm{IC}_{50}(n=3)$ was defined as the concentration of drug required to achieve a $50 \%$ reduction of enzymatic activity.

For the cleavage assay, a 5'-biotin-labeled 32-mer oligonucleotide (5'-CAGTGAGCGAGCTTCCGCTTGACATCCCA ATA-3'), which contains the strong topoisomerase site of mycobacterial TopoI, was used as substrate (Godbole et al., 2015). Reaction mixtures containing 1 unit of MtbTopoI in the same buffer as above were pre-incubated with SCN for $10 \mathrm{~min}$ at $4^{\circ} \mathrm{C}$. Then, $0.1 \mathrm{pmol}$ of the DNA substrate were added and the mix was incubated for $30 \mathrm{~min}$ at $30^{\circ} \mathrm{C}$. Reactions were terminated by adding $45 \%$ formamide dye and heating at $95^{\circ} \mathrm{C}$ for $5 \mathrm{~min}$. Samples were resolved on $12 \%$ denaturing $7 \mathrm{M}$ urea/PAGE using $1 \times \mathrm{TBE}$ as running buffer at $300 \mathrm{~V}$. DNAs were transferred to Hybond- $\mathrm{N}^{+}$membrane (Amersham) using the Trans-blot cell (Bio-Rad) in $1 \times$ TAE buffer according to the manufacturer's instructions and the blots were UVcross-linked. Detection was performed by autoradiography using chemiluminescent nucleic acid detection module from Thermo Fisher Scientific. Enzyme activity was determined by relative quantification of band intensities with ImageLab software.

\section{Activity of Alkaloids on DNA Supercoiling in $M$. tuberculosis Cultures}

Cultures of $50 \mathrm{ml}$ of $M$. tuberculosis H37Rv containing the plasmid vector pSUM36 were grown until $\mathrm{OD}_{600}$ of 0.4 was reached, which corresponded to a density of $1.7 \times 10^{7}$ bacterial cells $/ \mathrm{ml}$. The culture was split into three fractions, one of which was left untreated, while the other two were either treated with boldine at $500 \mu \mathrm{M}$ (corresponding to $1 \times \mathrm{MIC}$ ) or SCN at $15.6 \mu \mathrm{M}$ (corresponding to $1 \times \mathrm{MIC}$ ). After $4 \mathrm{~h}$ at $37^{\circ} \mathrm{C}$, total DNA was extracted from half of each fraction as described previously (Ferrándiz et al., 2010). At 24 h the other half of each fraction was processed as above. DNA samples were suspended in $30 \mu \mathrm{l}$ of distilled water and kept at $-20^{\circ} \mathrm{C}$ until analyzed in two-dimensional agarose gels.

\section{Analysis of the Topology of Covalently Closed Circles}

Circular DNA molecules were analyzed in neutral/neutral twodimensional agarose gels, a technique that allows to separate DNA molecules by mass and shape. The first dimension was run at $1.5 \mathrm{~V} / \mathrm{cm}$ in a $0.4 \%$ agarose (Seakem; FMC Bioproducts) gel in Tris-borate-EDTA (TBE) buffer for $17-19 \mathrm{~h}$ at room temperature. The second dimension was run at $7.5 \mathrm{~V} / \mathrm{cm}$ in $1 \%$ agarose gel in TBE buffer for $7-9 \mathrm{~h}$ at $4^{\circ} \mathrm{C}$. 
Chloroquine (Sigma) was added to the TBE buffer of both the agarose gel and the running buffer. After electrophoresis, gels were subjected to Southern hybridization. A 177-pb probe was obtained by PCR amplification of the kanamycinresistance gene of plasmid pSUM36 (Ainsa et al., 1996) with oligonucleotides PSUM36FBIOT 5'-TCGGCAGGAGCAA GGTGAGATGA-3' (biotinylated at $5^{\prime}$ ) and PSUM36R $5^{\prime}$-CC TGTCCGGTGCCCTGAATGAA-3'. This probe was used on the nylon membranes (Inmobylon $\mathrm{NY}^{+}$, Millipore) onto which the two-dimensional agarose gels had been transferred. Chemiluminescent detection of DNA was performed with the Phototope $^{\circledR}$-Star kit (New England Biolabs). Images were captured in a VersaDoc MP400 system and analyzed with the Quantity One program (Bio-Rad).

\section{RESULTS}

\section{Inhibition of $M$. tuberculosis Growth by Alkaloids}

We first tested the susceptibility of several M. tuberculosis isolates to boldine and its derivatives SCN and N-SCN (Table 1). In general, $M$. tuberculosis strains were more susceptible to boldine, SCN and $N$-SCN than what we reported previously for streptococci (data from García et al., 2011, are included in Table 1 for comparison). Susceptibility to boldine was low, with MIC values ranging from 31.25 to $>500 \mu \mathrm{M}$, in agreement with previous reports of the low anti-TB activity of this alkaloid (Guzman et al., 2010). Boldine derivatives SCN and N-SCN showed a much stronger antimicrobial activity, resulting in a minimum of a fourfold decrease in the MIC. For some clinical strains, such as HMS1292 (Table 1), the MIC of N-SCN was 32-times lower than that of the parent compound boldine, reaching as low as $1.95 \mu \mathrm{M}$, the lowest MIC we have detected. We speculated that sequence polymorphisms in the gene encoding MtbTopoI could explain the high susceptibility to alkaloids shown by some isolates, however, after sequencing Rv3646c alongside flanking regions in these strains, no polymorphisms were found. Hence, permeability, differences in the nucleoidassociated protein repertoire, or other unidentified mechanisms must be involved in basal susceptibility of mycobacteria to SCN and $N-S C N$.

\section{Cloning of the Gene Coding the DNA Topoisomerase I of $M$. tuberculosis H37Rv}

The MtbTopoI is encoded by topA gene (Rv3646c) in M. tuberculosis $\mathrm{H} 37 \mathrm{Rv}$ genome. The enzyme consists of 934 amino acids and has an estimated molecular weight of $102.3 \mathrm{kDa}$ (Cole et al., 1998). The topA gene was amplified by PCR from M. tuberculosis $\mathrm{H} 37 \mathrm{Rv}$ chromosomal DNA with specific oligonucleotides and cloned into the E. coli plasmid pQE-1 rendering plasmid pQE-MtbTopoI, which carries the Met(His) 6 -Gln-MtbTopoI fusion protein under the control of T5 promoter. This plasmid was introduced into E. coli XL1-Blue and its T5 promoter was induced by IPTG leading to overexpression
TABLE 1 | Susceptibilities of bacterial isolates to boldine and its alkaloid derivatives.

\begin{tabular}{llccc}
\hline \multirow{2}{*}{ Bacterial isolate } & & \multicolumn{3}{c}{ MIC $(\boldsymbol{\mu} \mathbf{M})$} \\
\cline { 3 - 5 } & & Boldine & $\mathbf{N - S C N}$ & SCN \\
\hline S. pneumoniae & R6 & 1000 & 16 & 16 \\
S. pneumoniae & ATCC 6303 & 1000 & 16 & 16 \\
S. mitis & NCTC 12261 & 1000 & 16 & 16 \\
M. tuberculosis & H37Rv & $>500$ & 15.6 & 15.6 \\
M. tuberculosis & GC 1237 & 31.25 & 7.8 & 7.8 \\
M. tuberculosis & HMS1553 & 31.25 & 1.95 & 3.9 \\
M. tuberculosis & HMS 1292 & 62.5 & 1.95 & 7.8 \\
M. tuberculosis & HMS1278 & $>250$ & 7.8 & 15.6 \\
M. tuberculosis & HMS 1498 & 31.25 & 3.9 & 7.8 \\
M. tuberculosis & HMS 1500 & $>250$ & 7.8 & 15.6 \\
M. tuberculosis & HMS 1536 & $>250$ & 7.8 & 15.6 \\
M. tuberculosis & HMS 1546 & $>250$ & 7.8 & 7.8 \\
\hline
\end{tabular}

of a protein with an apparent molecular weight of $100 \mathrm{kDa}$ (Figure 1A). This is in agreement with the expected size of the fusion protein $(102.3 \mathrm{kDa}$ for MtbTopoI $+1.23 \mathrm{kDa}$ for the N-terminal fusion). Although other proteins co-eluted with MtbTopoI (Figure 1B) they did not appear to interfere with the DNA relaxation activity of MtbTopoI on pBR322. The total yield of purification was $0.160 \mathrm{mg}$ of MtbTopoI per 21 culture. The final concentration was $8 \mathrm{mg} / \mathrm{l}$ and the recombinant MtbTopoI had a specific relaxation activity of $1.25 \times 10^{6}$ units/mg (see below).

To test the DNA relaxation activity of MtbTopoI, covalently closed negatively supercoiled (CCC) pBR322 plasmid (0.86 nM) was incubated in the presence of different amounts of the purified enzyme and the reaction products analyzed in monodimensional agarose gels as described (García et al., 2011). MtbTopoI activity converted the CCC plasmid into topoisomers with different degrees of supercoiling that were observed as discrete bands in a gel run in the absence of EtBr (Figure 1C). As expected, all relaxed topoisomers (RC) migrated as a single band when the gel was run in the presence of saturating concentrations of EtBr (Figure 1D). A unit of MtbTopoI enzymatic activity was defined as the amount of enzyme being able to relax $50 \%$ of the CCC substrate in $1 \mathrm{~h}$ at $37^{\circ} \mathrm{C}$. This corresponded to $8 \mathrm{ng}(3.6 \mathrm{nM})$ of purified enzyme.

\section{Inhibition of M. tuberculosis Topol Activity by Alkaloids}

We have previously reported inhibition of growth and inhibition of S. pneumoniae TopoI by 18 semisynthetic compounds derived from the natural alkaloid boldine (García et al., 2011). Out of these, two phenanthrene alkaloids, N-SCN and SCN, showed the greatest inhibition of $S$. pneumoniae growth. These were selected to be assayed for inhibition of MtbTopoI. While boldine did not inhibit the enzyme, even at concentrations up to $500 \mu \mathrm{M}$ (data not shown), MtbTopoI relaxation activity was inhibited in a concentration-dependent manner with $N$-SCN (Figure 2A), with $\mathrm{IC}_{50}$ values (average $\pm \mathrm{SD}$ ) of $5.6 \pm 0.8 \mu \mathrm{M}(n=3)$. 
A

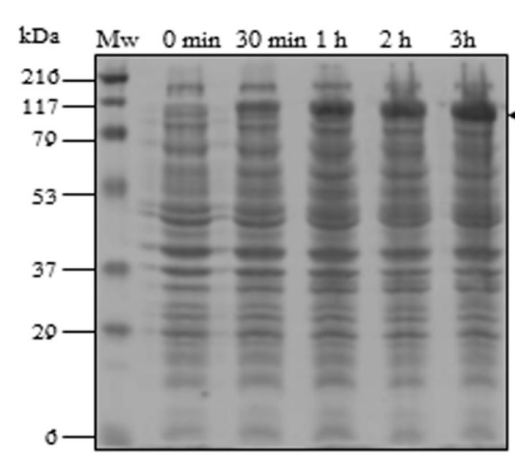

c

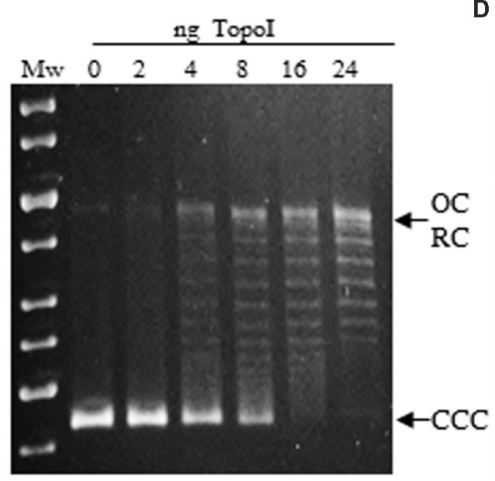

B

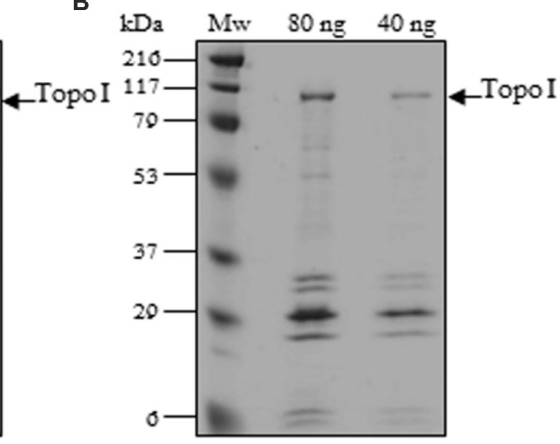

D

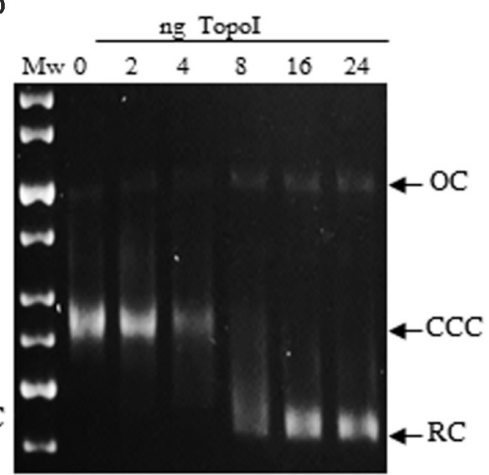

FIGURE 1 | Expression, purification and activity of Mycobacterium tuberculosis Topoisomerase I. (A) Cultures of Escherichia coli XL1Blue containing pQE1-MtbTopA were grown in LB medium and induced with IPTG at the indicated times. Crude extract samples of $10 \mu \mathrm{l}$ were electrophoresed in a SDS-12\% polyacrylamide gel. The polypeptides were visualized by Coomassie Blue staining. (B) Fractions extracted from a Ni-NTA column that showed topoisomerase I activity. (C,D) Plasmid pBR322 $(0.5 \mu \mathrm{g})$ was incubated with the indicated amounts of purified MtbTopol for $1 \mathrm{~h}$ at $37^{\circ} \mathrm{C}$. OC, relaxed open circles; CCC, covalently closed circles; RC relaxed circular plasmids forms. Mw, molecular mass standard. (C) Agarose gel run in the absence of EtBr. (D) The same samples as in C were run in the presence of $1 \mu \mathrm{g} / \mathrm{ml}$ of EtBr.

The inhibition by SCN was comparable, with $\mathrm{IC}_{50}$ values of $8.4 \pm 0.3 \mu \mathrm{M}(n=3)$ (Figure 2B). A good correlation between the inhibition of MtbTopoI activity and the inhibition of cell growth was observed for these compounds. Additionally, inhibition of cleavage of a 32-mer single-stranded DNA with SCN was detected (Figure 2C). Significant decreases $(P<0.05)$ of 1.7 - and 1.9 -fold (Figure 2D) in the amount of the 19-mer product were observed at $80 \mu \mathrm{M}$ SCN when compared with the untreated sample and $5 \mu \mathrm{M}$ treated samples, respectively. These results imply that MtbTopoI is indeed the in vivo target of SCN and N-SCN.

\section{Targeting of M. tuberculosis Topol in Vivo With $N$-SCN}

Evidence of the inhibition in vivo of MtbTopoI by $N$-SCN was obtained from the analysis of topoisomer distribution of the mycobacterial plasmid vector pSUM36 by two-dimensional agarose gel electrophoresis, a suitable approach for studying supercoiling levels (Ferrándiz et al., 2010). Treatment of pSUM36-containing $M$. tuberculosis cultures with $N$-SCN at $1 \times$ MIC resulted in a $20 \%$ increase of plasmid supercoiling (Figure 3). Supercoiling density $(\sigma)$ was -0.060 in the nontreated sample and in $1 \times$ MIC boldine-treated sample, and -0.072 in $1 \times$ MIC N-SCN-treated sample, indicating that plasmids became hypernegatively supercoiled. Although direct extrapolation from observations made in small plasmids is not completely equivalent to the bacterial chromosome, our results indicate that supercoiling significantly increases in the presence of $N$-SCN, and support the model that MtbTopoI is its target in vivo.

\section{Elucidation of the Mechanism of Action of SCN and $\mathrm{N}-\mathrm{SCN}$ in Mycobacteria}

Mycobacterium smegmatis was used as a model for determining the mechanism of action of SCN and N-SCN in mycobacteria. The MsPptrtopoI strain of M. smegmatis is a conditional knockdown strain which normally produces 1.5 less MsTopoI enzyme than wild type cells, and this level can be reduced further by 2.5 times in the presence of ATc (Ahmed et al., 2015). We observed that, accordingly to the reduction in the levels of MsTopoI enzyme, the MIC of SCN and N-SCN against M. smegmatis strains decreased (Table 2), whereas in contrast, the MIC of imipramine (a well-known topoisomerase poison; Godbole et al., 2015) increased. These experiments suggested that, since reduced levels of MsTopoI render the bacteria more susceptible to SCN and $N$-SCN, MsTopoI would be the target in vivo of these two inhibitors. 

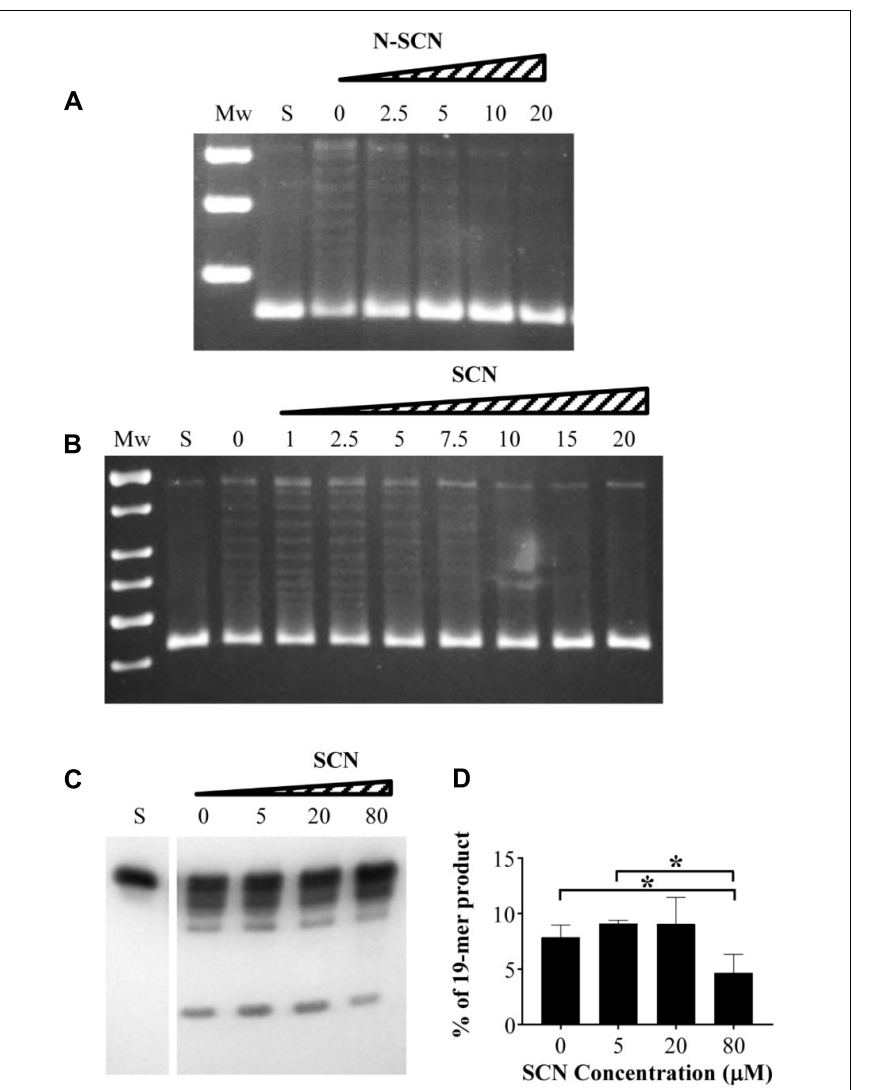

FIGURE 2 | Inhibition of $M$. tuberculosis topoisomerase I by SCN and N-SCN. (A,B) Supercoiled pBR322 $(0.5 \mu \mathrm{g})$ was treated with 1 unit of purified MtbTopol in $200 \mu$ l reactions containing N-SCN, SCN, or boldine at the concentrations $(\mu \mathrm{M})$ indicated. Mw, molecular mass standard; S, covalently closed supercoiled pBR322 used as substrate. (C) One unit of MtbTopol was pre-incubated with SCN at the indicated concentrations, then $0.1 \mathrm{pmol}$ of 5'-biotin-labeled 32-mer oligonucleotide was added and the reaction was continued for $30 \mathrm{~min}$. The products were resolved in 12\% denaturing PAGE. (D) Quantification of the 19-mer cleavage product. The values (mean \pm SD) of three independent experiments are shown. * indicates statistical significance by Student $T$-test $P<0.05$

In order to corroborate this finding, the kill time kinetics of inhibitory concentrations of $N$-SCN on $M$. smegmatis strains was determined after time. We compared the survival of the conditional knock-down mutant of M. smegmatis (MsPptrtopoI strain) with that of its corresponding complemented strain, which harbored a plasmid-encoded copy of the $M$. smegmatis topA gene. The complemented strain survived better than the parental one in the presence of 8-times the MIC of N-SCN, which can be attributed to a higher content of MsTopoI enzyme in the complemented strain (Figure 4).

\section{DISCUSSION}

The use of antimicrobials is one of the most effective ways to combat infectious diseases. In the case of $\mathrm{TB}$, effective drugs are scarce: these are just four first-line drugs used in the standard treatment of TB caused by drug susceptible strains.
Meanwhile, the incidence of drug resistant TB is increasing, despite the fact that the overall incidence of $\mathrm{TB}$ is decreasing slightly. This leaves a very limited number of therapeutic options. This situation has prompted investigation into new drugs, new targets, and new therapies for curing TB (Zumla et al., 2013, 2014). Natural products have been an extraordinary source of antimicrobials in general (Newman and Cragg, 2012; Cragg and Newman, 2013) and of anti-TB compounds in particular (Salomon and Schmidt, 2012). Natural products frequently lack some of the pharmacological properties desirable for a drug, making it necessary to develop semisynthetic products by chemical modifications. Boldine is a natural alkaloid produced by the tree Peumus boldus and has no significant antimicrobial activity. We have reported that two boldine derivatives (SCN and $N$-SCN) showed significant anti-pneumococcal activity (García et al., 2011). In this study, we have characterized the antimycobacterial activity of these drugs, along with the inhibitory activity on their potential target, the topoisomerase I enzyme.

Mycobacterium tuberculosis strains were susceptible to low concentrations of SCN or N-SCN. The reference strain H37Rv was the least susceptible strain, but even here the MIC was $15.6 \mu \mathrm{M}$ (equivalent to $5 \mu \mathrm{g} / \mathrm{ml}$ ). Other clinical isolates were susceptible at concentrations of $1.95 \mu \mathrm{M}$ (equivalent to $0.65 \mu \mathrm{g} / \mathrm{ml})$. Although these values are greater than the MICs of the most potent anti-TB drugs, isoniazid and rifampicin, they are in the same league as the MICs of ethambutol (another firstline antituberculosis drug) for strains susceptible to this drug (Christianson et al., 2014). The variation in the MICs among the various $M$. tuberculosis clinical strains could be related to their repertoire of nucleoid-associated proteins. For example, it has been shown that one of these proteins, HU, specifically regulates MtbTopoI activity (Ghosh et al., 2015). In any case, the range of MICs observed with SCN and N-SCN coincides with the $\mathrm{IC}_{50}$ values of the MtbTopoI enzyme (ca. $8 \mu \mathrm{M})$. This suggests that both SCN and N-SCN can efficiently cross the mycobacterial cell wall, which is a highly impermeable barrier that in general limits the in vivo activity of inhibitors shown to be potent enzyme inhibitors in vitro.

The comparison of MICs for S. pneumoniae (García et al., 2011) and $M$. tuberculosis showed that the latter is more susceptible to the Topo I inhibitors SCN and N-SCN. This fact could be related to the differences in the set of DNA topoisomerases available to each species. Both species have a single type I enzyme, but while $S$. pneumoniae possesses two type II enzymes (gyrase and topoisomerase IV), M. tuberculosis lacks topoisomerase IV (which carries out efficient DNA relaxation in addition to decatenation). This leaves gyrase as the only type II enzyme in M. tuberculosis (Manjunatha et al., 2002; Kumar et al., 2012). Altogether, this enzyme complement must maintain the appropriate level of chromosome supercoiling; in M. tuberculosis, gyrase carries out the dual function of both negative supercoiling and decatenation (Cole et al., 1998). Therefore, in the absence of topoisomerase IV, the only mycobacterial enzyme responsible for DNA relaxation is Topo I. Consequently, the inhibition of this enzyme by SCN and N-SCN would be more lethal in $M$. tuberculosis than in S. pneumoniae. Therefore, it would be worthwhile to investigate the antimicrobial activity of SCN 


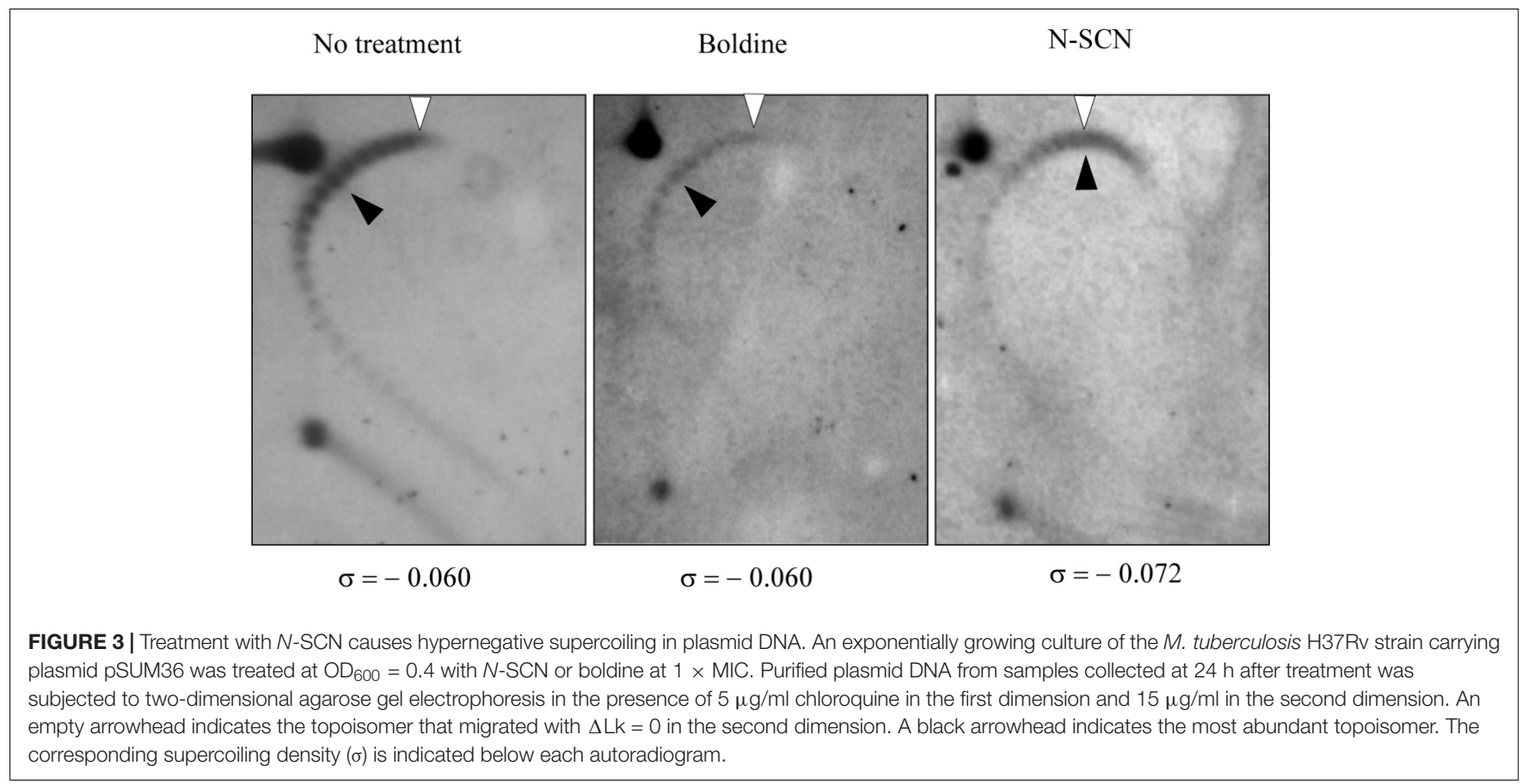

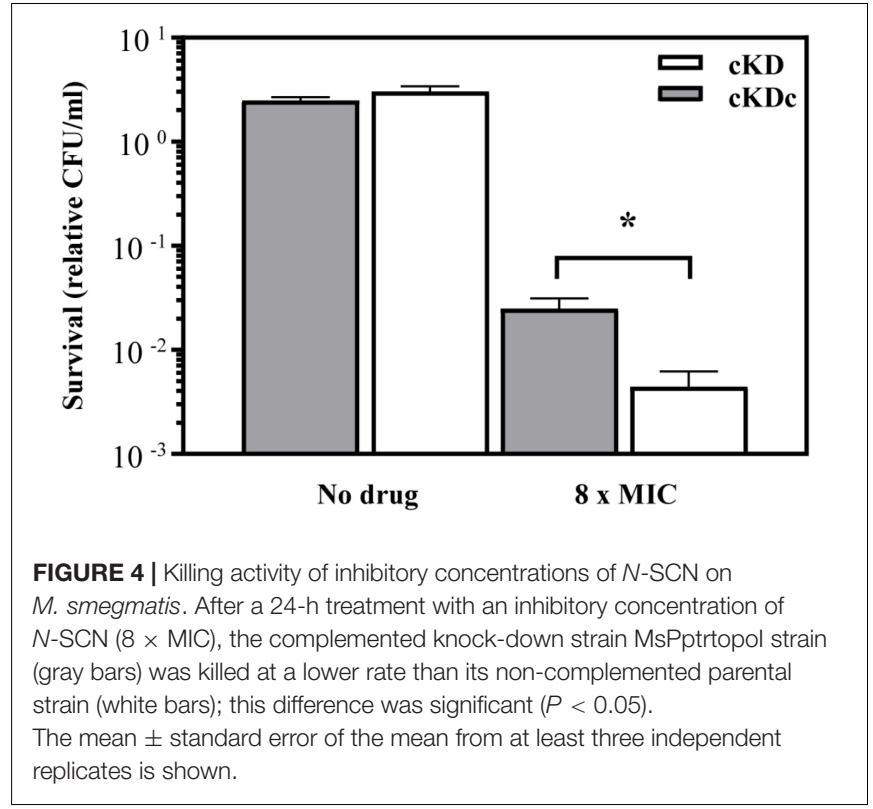

and N-SCN in other bacterial pathogens, which also lack topoisomerase IV, such as Helicobacter pylori (Debowski et al., 2012).

The topoisomer distribution of the plasmid pSUM36 under control conditions showed a mean superhelical density of -0.060 , a figure compatible with that reported for E. coli (Deng et al., 2005) and S. pneumoniae (Ferrándiz et al., 2010, 2016). Under treatment with $\mathrm{N}$-SCN at $1 \times$ MIC, a modest increase $(20 \%)$ in pSUM36 superhelical density was observed in M. tuberculosis. This value is lower than the $>58 \%$ observed due to treatment
TABLE 2 | Correlation between MICs of SCN and N-SCN and levels of MsTopol.

\begin{tabular}{|c|c|c|c|c|c|}
\hline \multirow[b]{2}{*}{$\begin{array}{l}\text { M. smegmatis } \\
\text { strain }\end{array}$} & \multirow[b]{2}{*}{$\begin{array}{l}\text { Levels of } \\
\text { Topol }\end{array}$} & \multirow[b]{2}{*}{ Imipramine } & \multicolumn{2}{|c|}{ MICs $(\mu \mathrm{M})$} & \multirow[b]{2}{*}{ Boldine } \\
\hline & & & $N-S C N$ & SCN & \\
\hline$m c^{2} 155$ & +++ & $125-200$ & 100 & $30-60$ & $>300$ \\
\hline MsPptrtopol & ++ & $175-300$ & 50 & $15-30$ & $>300$ \\
\hline MsPptrtopol + ATC & + & 350 & 25 & 15 & $>300$ \\
\hline
\end{tabular}

ATc, anhydrotetracycline was added at a concentration of $200 \mathrm{ng} / \mathrm{ml}$. Topoisomerase I levels in M. smegmatis strains: +++: wild type levels; ++: 1.5 times below wild type levels; +: 2.5 times below MsPptrtopol levels. Estimations according to Ahmed et al. (2015).

of S. pneumoniae with SCN at $1 \times$ MIC, but close to the $\pm 20 \%$ variation displayed during normal E. coli cell growth (Drlica, 1992). These results may indicate that, given the scarcity of the DNA topoisomerase complement of $M$. tuberculosis, the capacity of this bacterium to tolerate changes in supercoiling levels is more limited than with E. coli or S. pneumoniae.

The investigation of MtbTopoI as a drug target began very recently; when its requirement for cell survival was demonstrated (Ahmed et al., 2014). Until now, several MtbTopoI inhibitors have been discovered. The small molecule amsacrine, a eukaryotic type II topoisomerase poison used as an antineoplastic, is an inhibitor of this enzyme in $M$. tuberculosis (Godbole et al., 2014). Imipramine and norclomipramine are two drugs used clinically as antidepressants that also inhibit MtTopoI (Godbole et al., 2015). All these compounds are considered as topoisomerase poisons, since they perturb the cleavage-religation equilibrium and result in the accumulation of enzyme-DNA covalent adducts, which ultimately kill the bacterial cell (Nagaraja et al., 2017). There are other classes of compounds that directly 
inhibit TopoI (Sandhaus et al., 2016, 2018). SCN and N-SCN form part of this last class of compounds. Mechanistically, both types of inhibitors can be distinguished because increases in the levels of TopoI lead to a decrease in the MIC in the case of topoisomerase poisons, or to an increase in the MIC in the case of topoisomerase inhibitors (Nagaraja et al., 2017). In the case of SCN and N-SCN, the results we have obtained in this study using mycobacterial strains and in our previously published study with S. pneumoniae (García et al., 2011) indicate that they belong to the topoisomerase inhibitors class, not to topoisomerase poisons, since in both microorganisms we detected that increases in the quantity of TopoI corresponded with increases of their MICs. In fact, we showed that the inhibitory effect exerted by these alkaloids was enhanced prior to DNA binding, and in consequence, avoidance of DNA binding resulted in strong inhibitory effects (García et al., 2011).

Concerning toxicity, however, given the potent activity of amsacrine on mammalian TopoII, which results in cell death, it is unlikely that this compound could be used clinically against M. tuberculosis without further chemical modifications. We have reported that SCN and $N$-SCN only partially inhibited human TopoI at concentrations of $50 \mu \mathrm{M}$, well above the inhibitory concentrations we report in this work for both the enzyme and the bacterial cultures (García et al., 2011). In addition, SCN and $N$-SCN did not cause apoptosis of neutrophil even at $100 \mu \mathrm{M}$ and only slightly affected neutrophil survival at this concentration (García et al., 2011). In summary, the low toxicity of these compounds makes them very attractive leads for further anti-TB drug development.

\section{REFERENCES}

Ahmed, W., Menon, S., Godbole, A. A., Karthik, P. V., and Nagaraja, V. (2014). Conditional silencing of topoisomerase I gene of Mycobacterium tuberculosis validates its essentiality for cell survival. FEMS Microbiol. Lett. 353, 116-123. doi: 10.1111/1574-6968.12412

Ahmed, W., Menon, S., Karthik, P. V., and Nagaraja, V. (2015). Reduction in DNA topoisomerase I level affects growth, phenotype and nucleoid architecture of Mycobacterium smegmatis. Microbiology 161, 341-353. doi: 10.1099/mic.0. 000014

Ainsa, J. A., Martín, C., Cabeza, M., De la Cruz, F., and Mendiola, M. V. (1996). Construction of a family of Mycobacterium/Escherichia coli shuttle vectors derived from pAL5000 and pACYC184: their use for cloning an antibioticresistance gene from Mycobacterium fortuitum. Gene 176, 23-26. doi: 10.1016/ 0378-1119(96)00202-8

Cole, S. T., Brosch, R., Parkhill, J., Garnier, T., Churcher, C., Harris, D., et al. (1998). Deciphering the biology of Mycobacterium tuberculosis from the complete genome sequence. Nature 393, 537-544. doi: 10.1038/31159

Cragg, G. M., and Newman, D. J. (2013). Natural products: a continuing source of novel drug leads. Biochim. Biophys. Acta 1830, 3670-3695. doi: 10.1016/j. bbagen.2013.02.008

Champoux, J. J. (2001). DNA topoisomerases: structure, function, and mechanism. Annu. Rev. Biochem. 70, 369-413. doi: 10.1146/annurev.biochem.70. 1.369

Cheng, B., Cao, S., Vasquez, V., Annamalai, T., Tamayo-Castillo, G., Clardy, J., et al. (2013). Identification of anziaic acid, a lichen depside from Hypotrachyna sp., as a new topoisomerase poison inhibitor. PLoS One 8:e60770. doi: 10.1371/journal. pone.0060770

Cheng, B., Liu, I. F., and Tse-Dinh, Y. C. (2007). Compounds with antibacterial activity that enhance DNA cleavage by bacterial DNA topoisomerase I. J. Antimicrob. Chemother. 59, 640-645. doi: 10.1093/jac/dkl556

\section{AUTHOR CONTRIBUTIONS}

MG, DC, and JT-V performed the experiments related with cloning of the MtbTopoI gene in E. coli, protein expression, purification, and inhibitory assays in vitro. MF carried out the analysis of plasmid supercoiling. MA carried out the cleavage assay. LR and BG carried out the experiments with mycobacteria. JA and AC conceived, designed, supervised the study, and wrote the manuscript. All authors actively participated in the correction of the manuscript. The manuscript has been approved by all authors for publication.

\section{FUNDING}

This study was supported by grants BIO2017-82951-R (AC) and BIO-2009-09405 (JA) from the Plan Nacional of Ministerio de Economía y Competitividad, and grant 260872 (More Medicines for Tuberculosis) from the European Community's Seventh Framework Programme. CIBER de Enfermedades Respiratorias (CIBERES) is an initiative of ISCIII.

\section{ACKNOWLEDGMENTS}

We thank Tahl Zimmerman and Dessi Marinova for correcting the English version of the manuscript. We acknowledge V. Nagaraja for providing the M. smegmatis MsPptrtopoI strain and for helpful discussions on this manuscript.

Christianson, S., Voth, D., Wolfe, J., and Sharma, M. K. (2014). Re-evaluation of the critical concentration for ethambutol antimicrobial sensitivity testing on the MGIT 960. PLoS One 9:e108911. doi: 10.1371/journal.pone.010 8911

Debowski, A. W., Carnoy, C., Verbrugghe, P., Nilsson, H. O., Gauntlett, J. C., Fulurija, A., et al. (2012). Xer recombinase and genome integrity in Helicobacter pylori, a pathogen without topoisomerase IV. PLoS One 7:e33310. doi: 10.1371/ journal.pone.0033310

Deng, S., Stein, R. A., and Higgins, N. P. (2005). Organization of supercoil domains and their reorganization by transcription. Mol. Microbiol. 57, 1511-1521. doi: 10.1111/j.1365-2958.2005.04796.x

Drlica, K. (1992). Control of bacterial DNA supercoiling. Mol. Microbiol. 6, 425-433. doi: 10.1111/j.1365-2958.1992.tb01486.x

Ferrándiz, M. J., Martín-Galiano, A. J., Arnanz, C., Camacho-Soguero, I., TiradoVelez, J. M., and De la Campa, A. G. (2016). An increase in negative supercoiling in bacteria reveals topology-reacting gene clusters and a homeostatic response mediated by the DNA topoisomerase I gene. Nucleic Acids Res. 44, 7292-7303. doi: 10.1093/nar/gkw602

Ferrándiz, M. J., Martín-Galiano, A. J., Schvartzman, J. B., and de la Campa, A. G. (2010). The genome of Streptococcus pneumoniae is organized in topologyreacting gene clusters. Nucleic Acids Res. 38, 3570-3581. doi: 10.1093/nar/ gkq106

García, M. T., Blázquez, M. A., Ferrándiz, M. J., Sanz, M. J., Silva-Martín, N., Hermoso, J. A., et al. (2011). New alkaloid antibiotics that target the DNA topoisomerase I of Streptococcus pneumoniae. J. Biol. Chem. 286, 6402-6413. doi: 10.1074/jbc.M110.148148

Ghosh, S., Mallick, B., and Nagaraja, V. (2015). Direct regulation of topoisomerase activity by a nucleoid-associated protein. Nucleic Acids Res. 42, 11156-11165. doi: 10.1093/nar/gku804

Godbole, A. A., Ahmed, W., Bhat, R. S., Bradley, E. K., Ekins, S., and Nagaraja, V. (2014). Inhibition of Mycobacterium tuberculosis topoisomerase I by m-AMSA, 
a eukaryotic type II topoisomerase poison. Biochem. Biophys. Res. Commun. 446, 916-920. doi: 10.1016/j.bbrc.2014.03.029

Godbole, A. A., Ahmed, W., Bhat, R. S., Bradley, E. K., Ekins, S., and Nagaraja, V. (2015). Targeting Mycobacterium tuberculosis topoisomerase I by small-molecule inhibitors. Antimicrob. Agents Chemother. 59, 1549-1557. doi: 10.1128/AAC.04516-14

Guzman, J. D., Gupta, A., Evangelopoulos, D., Basavannacharya, C., Pabon, L. C., Plazas, E. A., et al. (2010). Anti-tubercular screening of natural products from colombian plants: 3-methoxynordomesticine, an inhibitor of mure ligase of Mycobacterium tuberculosis. J. Antimicrob. Chemother. 65, 2101-2107. doi: 10. 1093/jac/dkq313

Kumar, R., Madhumathi, B. S., and Nagaraja, V. (2014). Molecular basis for the differential quinolone susceptibility of mycobacterial DNA gyrase. Antimicrob. Agents Chemother. 58, 2013-2020. doi: 10.1128/AAC.01958-13

Kumar, R., Riley, J. E., Parry, D., Bates, A. D., and Nagaraja, V. (2012). Binding of two DNA molecules by type II topoisomerases for decatenation. Nucleic Acids Res. 40, 10904-10915. doi: 10.1093/nar/gks843

Manjunatha, U. H., Dalal, M., Chatterji, M., Radha, D. R., Visweswariah, S. S., and Nagaraja, V. (2002). Functional characterisation of mycobacterial DNA gyrase: an efficient decatenase. Nucleic Acids Res. 30, 2144-2153. doi: 10.1093/nar/30. 10.2144

Nagaraja, V., Godbole, A. A., Henderson, S. R., and Maxwell, A. (2017). DNA topoisomerase I and DNA gyrase as targets for TB therapy. Drug Discov. Today 22, 510-518. doi: 10.1016/j.drudis.2016.11.006

Newman, D. J., and Cragg, G. M. (2012). Natural products as sources of new drugs over the 30 years from 1981 to 2010. J. Nat. Prod. 75, 311-335. doi: $10.1021 / \mathrm{np} 200906 \mathrm{~s}$

Palomino, J. C., Martín, A., Camacho, M., Guerra, H., Swings, J., and Portaels, F. (2002). Resazurin microtiter assay plate: simple and inexpensive method for detection of drug resistance in Mycobacterium tuberculosis. Antimicrob. Agents Chemother. 46, 2720-2722. doi: 10.1128/AAC.46.8.2720-2722.2002

Palomino, J. C., and Martín, A. (2013). TMC207 becomes bedaquiline, a new anti-TB drug. Future Microbiol. 8, 1071-1080. doi: 10.2217/fmb.13.85

Salomon, C., and Schmidt, L. (2012). Natural products as leads for tuberculosis drug development. Curr. Top. Med. Chem. 12, 735-765. doi: 10.2174/ 156802612799984526

Sandhaus, S., Annamalai, T., Welmaker, G., Houghten, R. A., Paz, C., García, P. K., et al. (2016). Small-molecule inhibitors targeting topoisomerase I as novel antituberculosis agents. Antimicrob. Agents Chemother. 60, 4028-4036. doi: 10.1128/AAC.00288-16

Sandhaus, S., Chapagain, P. P., and Tse-Dinh, Y. C. (2018). Discovery of novel bacterial topoisomerase I inhibitors by use of in silico docking and in vitro assays. Sci. Rep. 8:1437. doi: 10.1038/s41598-018-19944-4

Sharifi-Rad, J., Salehi, B., Stojanović-Radić, Z. Z., Fokou, P. V. T., SharifiRad, M., Mahady, G. B., et al. (2017). Medicinal plants used in the treatment of tuberculosis - ethnobotanical and ethnopharmacological approaches. Biotechnol. Adv. doi: 10.1016/j.biotechadv.2017.07.001 [Epub ahead of print].

Snapper, S. B., Melton, R. E., Mustafa, S., Kieser, T., and Jacobs, W. J. (1990). Isolation and characterization of efficient plasmid transformation mutants of Mycobacterium smegmatis. Mol. Microbiol. 4, 1911-1919. doi: 10.1111/j.13652958.1990.tb02040.x

Tse-Dinh, Y. C. (2009). Bacterial topoisomerase I as a target for discovery of antibacterial compounds. Nucleic Acids Res. 37, 731-737. doi: 10.1093/nar/ gkn936

World Health Organization (2017). Global Tuberculosis Report. Available at: http: //www.who.int/tb/publications/global_report/en

Zumla, A., Nahid, P., and Cole, S. T. (2013). Advances in the development of new tuberculosis drugs and treatment regimens. Nat. Rev. Drug Discov. 12, 388-404. doi: 10.1038/nrd4001

Zumla, A. I., Gillespie, S. H., Hoelscher, M., Philips, P. P., Cole, S. T., Abubakar, I., et al. (2014). New antituberculosis drugs, regimens, and adjunct therapies: needs, advances, and future prospects. Lancet Infect. Dis. 14, 327-340. doi: 10.1016/S1473-3099(13)70328-1

Conflict of Interest Statement: The authors declare that the research was conducted in the absence of any commercial or financial relationships that could be construed as a potential conflict of interest.

Copyright (c) 2018 García, Carreño, Tirado-Vélez, Ferrándiz, Rodrigues, Gracia, Amblar, Ainsa and de la Campa. This is an open-access article distributed under the terms of the Creative Commons Attribution License (CC BY). The use, distribution or reproduction in other forums is permitted, provided the original author(s) and the copyright owner(s) are credited and that the original publication in this journal is cited, in accordance with accepted academic practice. No use, distribution or reproduction is permitted which does not comply with these terms. 